\title{
Smart Cities Simulation Environment for Intelligent Algorithms Evaluation
}

(

Pablo Chamoso Santos ${ }^{\text {a }}$, Fernando De La Prieta ${ }^{\text {b }}$

${ }^{\text {a }}$ Catholic University of Daegu, Republic of Korea.

b Sunchon National University, Republic of Korea.

chamoso@gmail.com

Smart Cities, Agents, Intelligent Systems
This article presents an adaptive platform that can simulate the centralized control of different smart city areas. For example, public lighting and intelligent management, public zones of buildings, energy distribution, etc. It can operate the hardware infrastructure and perform optimization both in energy consumption and economic control from a modular architecture which is fully adaptable to most cities. Machine-to-machine (M2M) permits connecting all the sensors of the city so that they provide the platform with a perfect perspective of the global city status. To carry out this optimization, the platform offers the developers a software that operates on the hardware infrastructure and merges various tech-niques of artificial intelligence (AI) and statistics, such as artificial neural net-works (ANN), multi-agent systems (MAS) or a Service Oriented Approach (SOA), forming an Internet of Services (IoS). Different case studies were tested by using the presented platform, and further development is still underway with additional case studies.

\section{Introduction}

The concept of Smart Cities (SC) is a current trend in technological projects. The balance with natural resources and the environment is responsible for these paradigms, which aim to increase the level of comfort for all citizens and institutions based on sustainable development.

When trying to make a city smarter, most of the efforts address energy areas because this will result in high resources saving which allow resources to be applied in other social areas. Therefore, improving the energy efficiency in cities is not only aims to reduce energy costs, but also to promote environmental and economic sustainability.

One of the main problems when developing hardware or software to increase citywide energy efficiency is the integration and deployment in real cities of a developed system, which can be tested and evaluated. In order to facilitate this integration task, a simulation environment that can test and evaluate both the hardware and the developed software or algorithms is presented.

SC can be represented as a three-level platform, where hardware represents the base, the communication mechanism represents the middle layer, and the top layer is represented by the intelligent software (Calvillo et al., 2016). 
The presented platform consists of a series of heterogeneous sensors that use M2M for the communication, and applies different AI and statistics technologies to evaluate the system and increase energy efficiency in cities.

One of the main characteristics of the simulation environment is that sensors can be easily integrated with the system. In fact, the most commonly used sensors have already been integrated and developers can use them to get the information. In addition, there are some open data services available to provide useful information that sensors cannot provide under simulated conditions, such as the city's weather forecast or traffic.

M2M communication platforms take the role of controlling the communication among all the connected elements of the cities. The decline in costs of connectivity and the price of required devices are driving the growth of this kind of communications. This platform is shaped by combining services to add some intelligence to these connected elements in an interoperable manner that provides the system with a highly heterogeneous content. That intelligence is added in the upper layer of the system to provide the best results depending on the environment status.

The rest of this paper is structured as follows: Section 2 shows the state of the art concerning projects and research conducted in the field of SC, showing the most commonly used techniques and technologies in this field, and then carrying out a comparison between them and the system presented. Section 3 presents the developed system, its operation and details of the techniques used. Section 4 describes the case studies developed to test the platform. Finally, Section 5 presents some results and conclusions of the work.

\section{Background}

The concept of SC or smart environments (Gómez-Romero et al., 2012) itself is still emerging. Making a city "smarter" is one of the main objectives of the researches as a strategy to reduce some problems caused by the rapid growth of the urban population. Problems such as pollution (visible in bigger cities), lack of resources, traffic congestion and deteriorating infrastructure are some of the many problems that large urban populations are increasingly facing (Chourabi et al., 2012).

There are multiple definitions of SC, for example as that proposed in (Washburn et al., 2009): "the use of smart computing technologies to make city services more intelligent, interconnected and efficient which includes administration, education, health care, public safety, real estate, transportation and utilities.”. From which we can deduce that new technologies (ICT, Information and Communication Technologies) are the base to provide better quality of life and prudent management of natural resources through the engagement of all citizens.

A smart city is also considered a "system of systems" (Naphade et al., 2011), where different integrated systems form a closed loop and are characterized by functions: sensing, information management, analytics, modeling, and influencing outcomes. Every system produces its own information and consumes the information of others in a well- defined urban planning.

More and more cities around the world are committed to developing pilot projects related to this movement, such as the SmartSantander ${ }^{\mathbf{1}}$ project in Santander, where there is a large display of parking

1 http://www.smartsantander.eu/ 
sensors that indicate available outdoor parking spaces to drivers. They have also deployed a municipal Wi-Fi network that aims to cover the entire city and obtain useful information about the number of Wi-Fi connections from all the parts of the city. Augmented reality applications focused on tourism are also offered. Málaga Smart City ${ }^{2}$ is a project that aims to save energy by applying micro power management, which consists of storing energy in batteries for use in buildings, street lighting and electrical transport. Another project is "Smart City Valladolid-Palencia"3. As the project is applied to two cities, and not one, it involves the additional task of addressing the issue of transport between the cities. The project has smart a meter network, integration of electric cars, energy efficiency in buildings, traffic organization, etc.

As previously mentioned, SC are typically composed of three levels: hardware, communication and intelligence. While this research work comprises all of them, its main focus is the first level, which addresses infrastructure and the way the information is gathered, but also includes some aspects analysis. The present study introduces a simulation environment that incorporates different technologies, techniques or algorithms and can be easily applied to any city.

With this platform, all innovations can be tested before deploying the infrastructure in a real city, which makes it possible to reduce the budget for the testing phase, allowing all cities to have their innovation impact evaluated and tested the with the platform before being deployed in the real city. The only requests to use the platform are: i) configure different settings about the simulated structures, ii) integrate the used sensor network following the platform rules, and iii) select different on-line open data services that will provide all the information that the algorithms require as input. The system offers the use of previously validated algorithms and simulation models to improve the energy efficiency: however, developers can define those to be used, and then compare them to choose the best option to use in the real city.

Regarding the hardware used for the simulation environment, the combined application of M2M along with a data preprocessing step help to consider the city as a homogeneous network of sensors, even though the sensors used are heterogeneous (Chamoso et al., 2015; Chamoso et al., 2016). The platform offers the use of different commonly used sensors to gather the most relevant information of any city, but new sensors can be integrated easily.

\section{Proposed architecture}

The proposed architecture is structured by following the previously identified three layer model as shown in figure 1 . To begin, the base of the architecture is represented by the city infrastructure, which is composed of every different objects of the city to be monitored in conjunction with the required sensor and the technology used, in order to exchange the data with the system.

Functionalities that are used to define and configure the sensor networks integrated in the objects are located in the middle layer. Due to the multiple kinds of objects that compose the network, communications are heterogeneous in nature. Consequently, it is necessary to pre-process the data to allow the system to use data independently of the technology used for the transmission, and thus allowing the system to be appreciated as a single and homogeneous network.

2 http://www.lacatedralonline.es/innova/system/Document/attachments/12351/original/IDCCiudadesinteligentes.pdf

3http://www.valladolidadelante.es/lang/modulo/?refbol=adelante-futuro\&refsec=smart-city-vyp\&idarticulo=79302 
The third and final layer is the associated to the intelligence of the city, where all the information is analyzed and managed in such a way that the highest benefits are provided and the most appropriate services are offered to the citizens.

The following section describes all the layers and their sub-systems.

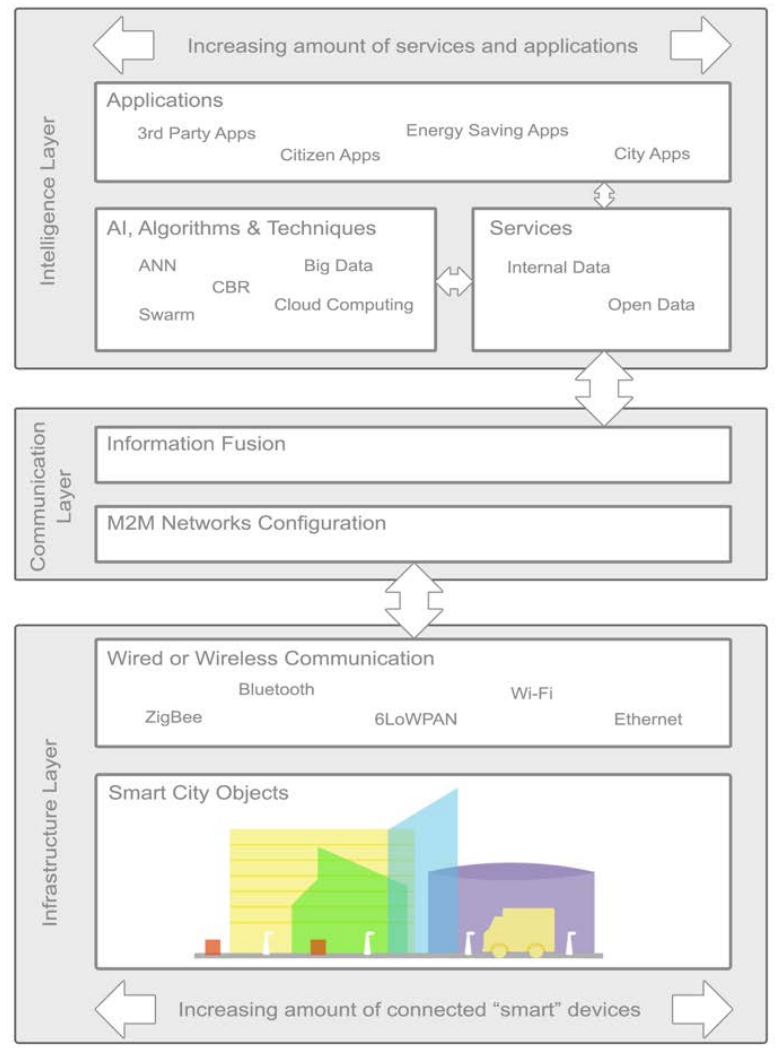

Fig. 1. System architecture overview

\subsection{Infrastructure Layer}

Starting from the lowest layer, the first level corresponds to both the connected objects of the city and the sensor networks.

The number of objects that can be connected to the network to be monitored as part of a global system is invaluable. Not only the number, but also the type of these connected objects grows substantially every day. The most common connected objects of smart cities were included in the developed simulation environment.

This simulation environment consists of a physical part, connected to a software part, so it differs significantly from the simulation systems used to date, which only include software simulations. Thus, with 
this kind of environment, multiple real sensors can be included and users can interact with the simulation environment, getting more realistic simulations.

The physical part is composed of different kinds of sensors and actuators and a city mockup, while all its elements were created with a 3D printer simulating real objects, buildings and cars. All these elements are connected in several different ways and they provide both real and simulated values. Real values are provided by the developers, who integrate their own prototypes with the mockup.

In addition, for every kind of element, the kind of technology that is more frequently used to gather or exchange information was analyzed and included as part of of the system. This ensures that widely applied technologies and techniques can be easily tested with the developed simulation environment.

The smart city common objects that have already been embedded in the simulation environment are:

Buildings: there are different kind of buildings distributed among the city mockup, each of which is organized in different districts according to its category. For example, there are residential, business, commercial, industrial or energetic districts. Each has its own features, for example when consuming or generating energy. These values can be simulated or provided by the developers when testing the system. The simulated values are based on different parameters like the day, the current time or the configured energetic infrastructures.

Public lighting: a series of lamps was distributed along the streets of the simulation environment. The system makes it possible to both simulate and analyze the public lighting to detect failures and find optimal solutions. Moreover, the system allows the testing of different energy distribution algorithm according to different parameters, such as the assigned budget or traffic.

Containers: there are several groups of recycling and waste containers distributed among the city districts. Their value can be simulated according to the consumption habits of every district or even directly associated to real sensors that measure the fill level of the containers. Different calculations can be evaluated by using those data. For example, the route of every track can be optimized to collect only the waste of the full containers, or to change the location of those containers that are always empty.

Energy generation elements: several elements that simulate the production of electric power from the sun or the wind were included in the simulation environment. Data are simulated by using real open data services of the city that it is being simulated.

Vehicles: one of the most important aspects to bear in mind when trying to make a city smarter is its traffic management and distribution. Vehicles, whether public or not, play an important role when evaluating the quality of the lives of the citizens and that of the environmental, which is often harmed due to high pollution rates. Accordingly, all the vehicles included in the simulation environment are monitored and controlled to carry out different simulations. In order to guide them among the roads of the city, a steel rail is embedded in a methacrylate base and covered by a vinyl that simulates the road. Junctions are possible as a result of the servomotors that move the rail to follow the desired direction. Front wheels are guided by a small magnet that follows the steel rail, and rear wheels are directly connected to a DC motor whose speed can be controlled by software. To know the position of every car, several magnets were embedded in parallel to the steel rail, forming binary patterns that identify the street. To detect those magnets, cars are equipped with a hall sensor, allowing their position to be known at all times.

The global sensor network may be composed of multiple heterogeneous sets of individual sensors associated to one or more objects of the city. These sensors are in charge of automatically gathering the requested data of the nearest environment by themselves. 
Table 1 identifies the sensors that were adapted to the main common objects, and the communication methods used in the test.

\begin{tabular}{|l|l|l|}
\hline \multicolumn{1}{|c|}{ Elements } & \multicolumn{1}{c|}{ Sensors } & \multicolumn{1}{c|}{ Communications } \\
\hline Buildings & Ammeter, temperature, humidity & Serial*, Wi-Fi, Ethernet \\
\hline Lamps & Ammeter, voltmeter, LDR, $\mathrm{CO}_{2}$ & PWM*, Wi-Fi, Ethernet \\
\hline Containers & Ultrasonic, temperature & Wi-Fi, BLE, 4G, GSM \\
\hline Solar panels & Ammeter, voltmeter & 4G, GSM \\
\hline Vehicles & Hall*, voltmeter, ammeter & BLE, Wi-Fi \\
\hline
\end{tabular}

Table 1. Mockup connected elements, built-in sensors and the communication type.

Sensors and communications marked with an asterisk $(*)$ are only used for their integration with the simulation environment.

\subsection{Communication Layer}

Sensors must also incorporate some kind of mechanism to transmit this information so that it can be integrated in the global system for future use. On many occasions, the cities already use sensor networks that have been incorporated in order to develop studies or to take measures. They could also have been added due to some other reason, prior to the process of transforming the city into a smart city. In addition to the previously commented evolution in technology, this could result in the cohabitation of different kinds of sensors, gathering the information associated to a same environment.

Large-scale systems typically include middleware (MW) to collect information coming from the sensors. The main task of this MW is to allow the information gathered by the sensors to be standardized within the system. To do so, the system includes an intermediate layer specialized in collecting information by using the required protocol for each technology. Once the information is obtained by the sensor and transmitted, the MW collects it and processes it (without interruption) to produce a new encapsulation to a common protocol for the whole system.

This way, the inclusion of new technologies, which may appear in the future to offer new or better features, prevents the bulk of the smart-cities management system from being affected in the case of using them. In such cases, the procedure would be based, as a general rule, on creating a new module incorporating the MW as a driver. This module will include the programming associated to the conversion of information formats and communication protocols. In turn, it will allow the definition of every configuration parameter that such technology requires.

The inclusion of an MW with such characteristics is necessary in the system, as it means a reduction in risk of some factors that affect the conflict of decision making regarding the technology to be used in a city. In particular, the communication protocol directly affects important factors such as sustainability, adaptability and system maintenance over time.

Hosting and information processing services are performed in a Cloud Computing (CC) environment; therefore, such environments support the communication and subsequent levels. The application of CC technology, particularly to smart city management, provides a deployed system with different benefits. To begin, the initial investment will be reduced due to the fact that there is no need to acquire hardware and software to deploy the system. Flexibility is the other main advantage provided by CC because the service 
is capable of adapting itself to the demand and always providing the necessary resources. As a result, the size of the city is no longer relevant.

Using a practical case taken from this work as an example, the MW is divided into a series of modules, each of which is associated to a different technology. For example, to communicate through Bluetooth 4.0 (BLE - Bluetooth Low Energy), there is a module in charge of requiring the technology-specific information needed to register a sensor of this kind. For the case of BLE, this information only includes the MAC (Media Access Control) address. Once the configuration has been stored, the Gatttool library is internally used. This library makes it possible to use any BLE device through a series of simple commands. Similarly, there are specific modules to communicate through ZigBee, RFID or Wi-Fi technology, as well as through wired sensors, independently of their protocol or API. In every particular case, the specific deencapsulation, associated to the technology, is performed. To do so, the obligatory information is extracted and the encapsulation to the protocol required by the higher layers in the system is performed. These processes are all performed remotely (in the CC environment), which allows us to take advantage of the benefits each technology offers regarding consumption, range or duration, without affecting the gathering of data by the higher levels of the architecture.

Thus, it is necessary to include a MW with such features in the system, as it implies the resolution of the protocol incompatibility conflict, which directly affects the stability of the entire system. However, despite using these techniques, additional conflicts still remain.

\subsection{Intelligence Layer}

The last layer is composed of three big blocks: i) the set of technics and algorithms used for the data treatment and processing; ii) the generation of several services based on the platform data or the integration of third party services to allow real simulations; iii) the set of final applications.

First, the set of technics and algorithms implemented in the platform is scalable and reusable. So far, there are different technics and algorithms that allow developers to store, manage and analyze all the system data. As the number of connected objects can grow, the storage infrastructure and the data treatment algorithms must be ready to grow at the same pace. The last layer is deployed in a CC platform and uses mechanisms to deal with big data.

Developers that use the platform can specify their own AI algorithms or use those that have been proposed to test them or compare their effectiveness.

To use the proposed algorithms, the platform architecture must be based on SOA. A set of internal and third party data based services are offered, which the developers are free to access because of the IoS.

These services are used by all the developed applications, which increase the quality of services delivered to the citizen or to the city in general. For example, the simulation environment provides different apps such as citizen recommendation, citizen participation, energy management, efficiency measurement or public services path planning.

\section{Results, Conclusions and Future Work}

Different vertical applications have been developed and integrated with the simulation environment. This article is focused on the development of a platform that can optimize various aspects of city management, 
including the collection routes used by waste trucks. In this case, the optimization is performed by taking into consideration the fill level of the glass, paper, plastic and waste containers of the city.

This section details the case study, specifically the hardware integration with the simulation environment platform, which is the lower layer, as the upper layer is designed for the applications of other developers. Finally, conclusions and future work are presented.

\subsection{Waste Collection Optimization}

As previously mentioned, the mockup is composed of different districts with different containers: paper, glass, plastic and waste. During simulation, they are filled in a pseudo-random way, taking into account different parameters associated to their district, the time or the weekday of the simulation.

Two different kinds of sensors were integrated, both of which are associated to the value of two mockup containers. Different tests to place the sensor in a real container were evaluated, as well as the type of communication.

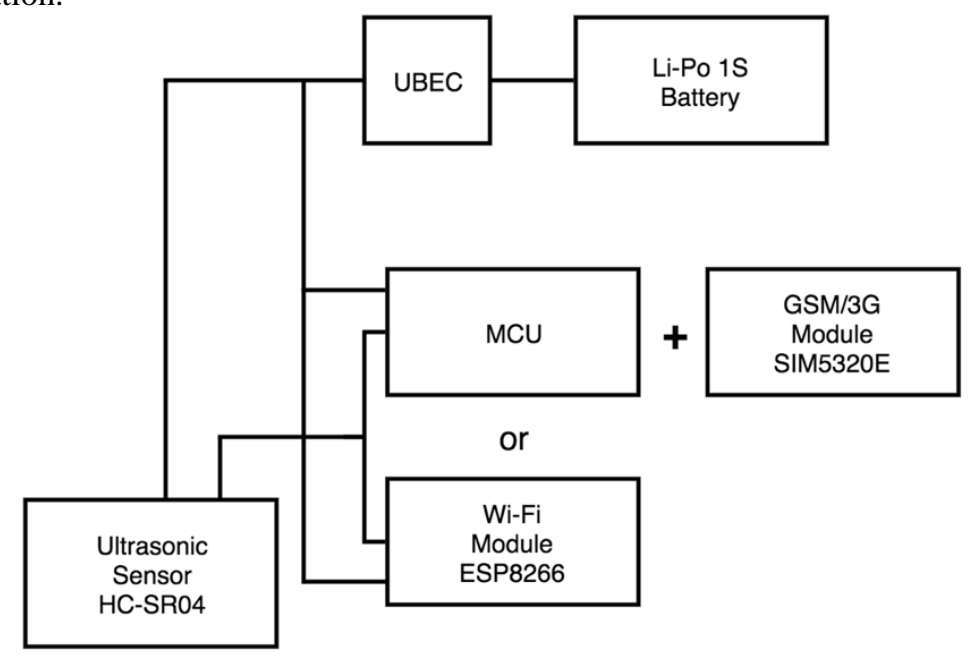

Fig. 2. Waste Level Sensor Schema.

Figure Fig. 2 shows the schema of the sensor used for the integration with the two evaluated communication types: i) by using a GSM 3G module, which requires the use of a microcontroller to analyze the measured data, and ii) a Wi-Fi module with integrated processing capacity, which is capable of reading the measured value, processing and transmitting it only when there is a change, which saves the battery life.

The sensor used in this case study is an HC-SR04, which works at a frequency of $40 \mathrm{kHz}$ in a range from $1.7 \mathrm{~cm}$ to $4.5 \mathrm{~m}$, thus making it very suitable for use in containers.

In order to consider a measured value as different from the previous one, the difference must be more than 2 centimeters and the change must last a minimum of 10 seconds. This results in a considerable saving of energy and the tested battery, with a capacity of 3200mAh can last up to 26 days.

Over this infrastructure and communication layers, different algorithms for optimizing the path taken by the waste trucks were been evaluated. As the positions of the trucks are also known by the system, only those containers with a level higher than $50 \%$ are included in the route. 


\subsection{Conclusions and Future Work}

This physical simulation environment of smart cities, completely integrated with a software platform, is unlike other simulation systems that only offer software simulations.

The main advantage of this work is the possibility of including physical simulation tests, which are more complete than mere software simulations.

Given the presented case, in which volumetric sensors were placed inside the containers of the city, the company responsible for the recycling would benefit from cost savings with both personnel and resources, as only one set of containers would be included in the truck route.

As future work, a virtual organization based multi-agent system will be included in order to organize the platform autonomously in line with the requirements of the city's evolution, regardless of the city characteristics or values.

\section{Acknowledgements}

The research of Pablo Chamoso has been financed by the Regional Ministry of Education in Castilla y León and the European Social Fund (Operational Programme 2014-2020 for Castilla y León, EDU/310/2015 BOCYL) and the project EKRUCAmI: Europe-Korea Research on Ubiquitous Computing and Ambient Intelligence. Ref. 318878. FP7-PEOPLE-2012-IRSES.

\section{References}

Calvillo, C. F., Sánchez-Miralles, A., \& Villar, J. (2016). Energy management and planning in smart cities. Renewable and Sustainable Energy Reviews, 55, 273-287.http://dx.doi.org/10.1016/j.rser.2015.10.133

Chamoso, P., De la Prieta, F., De Paz, F., \& Corchado, J. M. (2015). Swarm Agent-Based Architecture Suitable for Internet of Things and Smartcities. In Distributed Computing and Artificial Intelligence, 12th International Conference (pp. 21-29). Springer International Publishing. http://dx.doi.org/10.1007/978-3-319-19638-1_3

Chamoso, P., De la Prieta, F., Bajo, J., \& Corchado, J. M. (2016). Conflict Resolution with Agents in Smart Cities. Interdisciplinary Perspectives on Contemporary Conflict Resolution. IGI Global. http://dx.doi.org/10.4018/978-1-5225-0245-6.ch014

Chourabi, H., Nam, T., Walker, S., Gil-Garcia, J. R., Mellouli, S., Nahon, K., Pardo, T. A., and Scholl, H. J. (2012). Understanding Smart Cities: An Integrative Framework. 2012 45th Hawaii International Conference on System Sciences, 2289-2297. http://dx.doi.org/10.1109/HICSS.2012.615

Gómez-Romero, J., Serrano, M. A., Patricio, M. A., García, J., \& Molina, J. M. (2012). Context-based scene recognition from visual data in smart homes: an information fusion approach. Personal and Ubiquitous Computing, 16(7), 835-857. http://dx.doi.org/10.1007/s00779-011-0450-9

Naphade, M., Banavar, G., Harrison, C., Paraszczak, J., \& Morris, R. (2011). Smarter cities and their innovation challenges. Computer, 44(6), 32-39. http://dx.doi.org/10.1109/MC.2011.187

Washburn, D., Sindhu, U., Balaouras, S., Dines, R. a, Hayes, N., and Nelson, L. E. (2009). Helping CIOs Understand "Smart City" Initiatives. Growth, 17. Available at: http://c3328005.r5.cf0.rackcdn.com/73efa931-0fac-4e28-ae77-8e58ebf74aa6.pdf.. 

